\title{
A Heuristic Operator-Based Quantum Ant Colony Optimization Algorithm for Robot Path Planning
}

\author{
Xiaoming You ${ }^{1}$, Sheng $\mathrm{Liu}^{2}$ and Chen Zhang ${ }^{1}$ \\ ${ }^{1}$ College of Electronic and Electrical Engineering, Shanghai University of \\ Engineering Science, No. 333, Longteng Road, Shanghai, 201620, China \\ E-mail:yxm6301@163.com \\ ${ }^{2}$ School of Management, Shanghai University of Engineering Science, China, \\ 201620 \\ E-mail:ls6601@163.com
}

\begin{abstract}
A Heuristic operator-based Quantum ACO algorithm (HQACO) to solve the global robot path planning problem is presented. The idea of the algorithm is that first an ant colony algorithm based on quantum computing is proposed to plan the optimal collisionfree path. Then a new heuristic operator which balances between population diversity and convergence rate is defined. Heuristic roulette selection strategy is adopted to avoid the search being trapped in local optimum. We redefine the heuristic operator to guide the algorithm converge fast. And the Quantum gate (Q-gate) is used to update the pheromone in ACO in order to increase the diversity of population. Simulation results show that HQACO not only enhances the global search capability, but also more effective than the path planning method in the literature, We demonstrate also that the novel ACO algorithm can find more precise solutions for path planning problems under the complex environment.
\end{abstract}

Keywords: Global Path Planning, Ant Colony Optimization, Heuristic Roulette. Quantum Computing

\section{Introduction}

Robotic is an essential part in manufacturing processes automatization. And robotic is gaining a lot of interest in our daily life and in several areas including industrial applications, manufacturing and constructions, search, rescue, environment exploration and others [1]. Mobile robots path planning is a critical and fundamental problem in the field of mobile robots, whose main task is finding a collision-free path for the robot starting from its initial location to the target location in a given environment with obstacles while satisfy a set of optimization criteria including traveled distance, processing time and energy consumption. The grid model is abstract model of mobile robots path planning environment. It is directly and easy to establish model, so it has been widely used. There are many methods based on the grid model, such as: freedom tree, Genetic Algorithm, ACO-grid [13].

During the past years, A number of path planning algorithms such as $\mathrm{A}^{*}$, PSO and ACS [1] have been proposed for use in mobile robots. However, those methods are not enough efficient, poor global search capability, and not enough adaptability to complex spatial domain. An improved ant colony optimization [3] has drawn more and more attention from researchers and has been widely used in a variety of applications, including network routing [3], traveling salesman [2], quadratic assignment [4], and mobile robot path planning problem [6-13]. Many scholars have conducted extensive research to improve ant colony algorithms global search capability. However, the ant colony 
algorithm is also easy to get trapped in a local optimum for robot path planning problem. The crux is the balance and compromise between population diversity and convergence rate, which can prevent falling into the local optimum or escape from it.

This paper is to address the problem of robot path planning using Improved $A C O$ algorithm. Improved operator including heuristic operator and quantum computing, heuristic operator would balance between population diversity and convergence rate, quantum computing would increase the population diversity. It can also quickly find an optimal path planning under the complex environment.

First, we adopt grid modeling to establish the environment model, and then we use the modified ACO algorithm to obtain the global optimal path. In the modified ACO algorithm, a Quantum gate (Q-gate) is used to update the pheromone in ACO in order to increase the diversity of position and a new heuristic operator which balances between population diversity and convergence rate is defined. It complements the modified ACO to avoid getting trapped in the local optimum and improve the solution quality. We describe the improved performance of HQACO. The experiment results show that the performance of the HQACO algorithm clearly outperforms algorithm in the literature and it is an effective algorithm for robot path planning under Grid Modeling environment.

\section{Related Works}

The first ACO algorithm, called Ant System (AS), was applied to the Traveling Salesman Problem (TSP) by Dorigo M. [1]. It constitutes the main framework of other ACO algorithms and is considered as a prototype. Ant colony system (ACS) is one of the most successful ACO algorithms which achieve a much better performance than ant system (AS). In ACS, the most interesting thing is the transition rules to ants' searching solutions. When an ant chooses the next nodes, it no longer completely obeys the previous experience, but it has a certain probability to choose the shorter routers with higher pheromone intensity. It is called pseudorandom proportional rule, defined by Dorigo and Stülzle [3] as:

$$
j=\left\{\begin{array}{cc}
\arg \max \left\{\left[\tau_{i l}\right]\left[\eta_{j l}\right]^{\beta}\right\}, & q \leq q_{0} \\
J\left(p_{i j}^{k}\right), & q>q_{0}
\end{array}\right.
$$

Where $q$ is a random variable uniformly distributed in $[0,1](\mathrm{q} \in[0,1]), q_{0}$ is a parameter according to the previous empirical values used in this case for the best possible move $\left(q_{0} \in[0,1]\right), k$ is an ant, $i, j$ are the initial and the next node, $l$ is a candidate solution that ant $k$ have not travel the node in a loop, $J$ is a random function gained from the probability distribution $p_{i j}^{k}$ (see equation 2 ), and it will be chose by the ant $k$ if $q>q_{0}$.

$$
p_{i j}^{k}=\frac{\left[\tau_{i j}\right]^{\alpha}\left[\eta_{i j}\right]^{\beta}}{\sum_{l \in N_{i}^{k}}\left[\tau_{i j}\right]^{\alpha}\left[\eta_{i j}\right]^{\beta}}
$$

Parameter $\beta$ determines the relative influence of the heuristic information, $\eta_{i j}$ $\left(\eta_{i j}=\frac{1}{d_{i j}}\right)$

node $i$ and $j N_{i}^{k}$ is the set of candidate nodes connected to node $i$, with respect to ant $\mathrm{k}$. $\tau_{i j}$ represents the pheromone from node Error! Reference source not found. to node Error! Reference source not found., After constructing its tour, only the ant to find the 
shortest path to the current loop was allowed to update the pheromone by applying the pheromone updating rule equation (3), which accelerates the convergence of the algorithm to some extent.

$$
\begin{gathered}
\tau_{i j}(\mathrm{t})=(1-\varphi) \tau_{i j}(\mathrm{t})+\varphi \Delta \tau_{i j}(\mathrm{t}) \\
\Delta \tau_{i j}=\sum_{k=1}^{n} \Delta \tau_{i j}{ }^{k}
\end{gathered}
$$

With $\varphi$ in $(0,1)$, and $\Delta \tau_{i j}(\mathrm{t})$ is the quantity of pheromone deposited by the ant $\mathrm{k}$ between time ${ }^{t}$ and $t+1$ on the edge $(i, j)$. In addition to the global update rule, ACS uses the ant-cycle updating rule. An ant-cycle system information update model is

$$
\Delta \tau_{i j}{ }^{k}=\left\{\begin{array}{cc}
\frac{Q}{L_{k}}, & \operatorname{arc}(\mathrm{i}, \mathrm{j}) \text { belong to best loop } \\
0, & \text { otherwise }
\end{array}\right.
$$

represents the intensity of the pheromone, it affects the convergence speed of algorithm to some extent, ${ }^{L_{k}}$ denotes the total length that the ant $k$ passed in this cycle.

At present, numerous methods to solve the robot path planning have been attempted. For instance, in [6] the author proposed an ant algorithm based on rolling planning for the mobile robot path planning problem in an environment where the global information was unknown. In [7], the authors presented a differential evolution chaos ant colony optimization (DEACO) algorithm to plan the optimal collision-free path for a mobile robot in a complicated static environment. Simulation results indicated that an optimal and safe path which the robot moved on can be rapidly obtained even in a complicated geographical environment. In [8], the authors utilized the locations of start and goal to build the environmental model based on the simplified visibility graph. In their algorithm, the local path information was integrated with the initialization of pheromone and the selected probabilities of the paths, resulting in improving the convergence speed and avoiding the premature phenomenon as far as possible. In [10], the modeling method based on Agent was introduced to construct the ant colony foraging behavior model. The simulation results showed the ants foraging behavior model can quickly determine the optimal path in the complex environment. In [12], the authors proposed the Best-Worst Ant System, the simulation shows that the robot can avoid the obstacle smoothly and can find an optimal path quickly, but the process is too complicate, even not avoid the path deadlock problem completely. In [13], the authors proposed a new optimal algorithm (ACO-grid), in which there is a heuristic based on the distance from the position to the goal, the times that the position has been visited and the moving direction of robot. ACOgrid can be verified to be effective by the computational result in the test instances. But because of the pheromone update too slowly to make the ant's objective not clear on path selection, so the convergence time is too long.

Some other research efforts have studied the problem of mobile robot path planning under dynamic environment. For instance, Wang Zhe et al introduced the artificial potential field concept which made ants more sensitive to the optimal path into the ant colony algorithm during the global searching process [9]. The mobile robot carried out different obstacle avoidance strategies respectively when different types of obstacle appear in the dynamic environment.

Quantum computing is a very attractive research area. Due to its unique computational performance, the quantum computing has attracted extensive attention of researchers. Some studies have used quantum ACO algorithm in solving robot path planning [14]. Quantum evolutionary algorithm takes advantages of a dynamic balance between diversification and intensification. It can be equipped with additional mechanisms and 
operators which improve the quality of obtained results. The quantum gate (Q-gate) can be used to update the pheromone in ACO; the quantum gate adjustment strategy is as follows:

$$
\left(\begin{array}{c}
\alpha_{i}^{\prime} \\
\beta_{i}^{\prime}
\end{array}\right)=\left(\begin{array}{l}
\cos \left(\theta_{i}\right)-\sin \left(\theta_{i}\right) \\
\sin \left(\theta_{i}\right) \cos \left(\theta_{i}\right)
\end{array}\right)\left(\begin{array}{l}
\alpha_{i} \\
\beta_{i}
\end{array}\right)
$$

\section{The Heuristic Operator-Based Quantum ACO Algorithm (HQACO)}

Modified ACO algorithm phase: It is executed in eight steps.

Step 1. Initialization populations $Q_{k}(0)$ of the Quantum ACO, set the iteration number $i=0$.

$$
Q_{k}(0)=\left\{q_{k 1}^{0}, q_{k 2}^{0}, q_{k 3}^{0}, \ldots, q_{k n}^{0}\right\}, k=1,2, \ldots m
$$

Step 2. Put the starting point into $t a b u_{k}$, choose one subsequent node $j$ to move according to

$$
\begin{gathered}
j=\left\{\begin{array}{cc}
\arg \max \left\{\left[\tau_{i l}\right]\left[\eta_{j l}\right]^{\beta}\right\}, & q \leq q_{0} \\
J\left(p_{i j}^{k}\right), & q>q_{0}
\end{array}\right. \\
q_{0}=q_{0} \times \frac{n c}{N C} \times \theta
\end{gathered}
$$

$q_{0}$ is a heuristic roulette selection parameter, $n c$ is the number of iterations, $N C$ is the max number of iterations, $\theta$ is the accelerating convergence factor. Finally put node $j$ into ${ }^{t a b u} u_{k}$.

Step 3.Loop (Step 2) until each ant finds the target point, calculate the tour length ${ }^{L_{k}}$ of ant $k$, and record the current optimal solution.

Step 4.Update the pheromone intensity of paths which is shorter than $L_{i}$ (it has already set in this work) by equation (3).

Step 5.The number of iterations plus 1 .

Step 6.If the number of iteration reach the maximum number of iterations and find the same solutions, output the best solution.

Step 7.Use quantum gate equation (6) to update pheromone intensity on some paths.

Step 8.To clear the ${ }^{t a b u_{k}}$ of each and move to (Step 2)

End.

\section{Experimental Analysis}

In this section, HQACO was implemented in matlab7.0, and the tests were performed on a $400 \mathrm{MHz}$ UltraSparc2 processor. We mainly examined the impact of modified operator on the solution quality and validity characteristics of the algorithm. In our computational experiment, the model was tested with multiple grid data, including the 20 $\times 20,50 \times 50$ and more. We presented a performance evaluation study of the proposed HQACO algorithm applied to complex environments [13] illustrated in Figures1. 

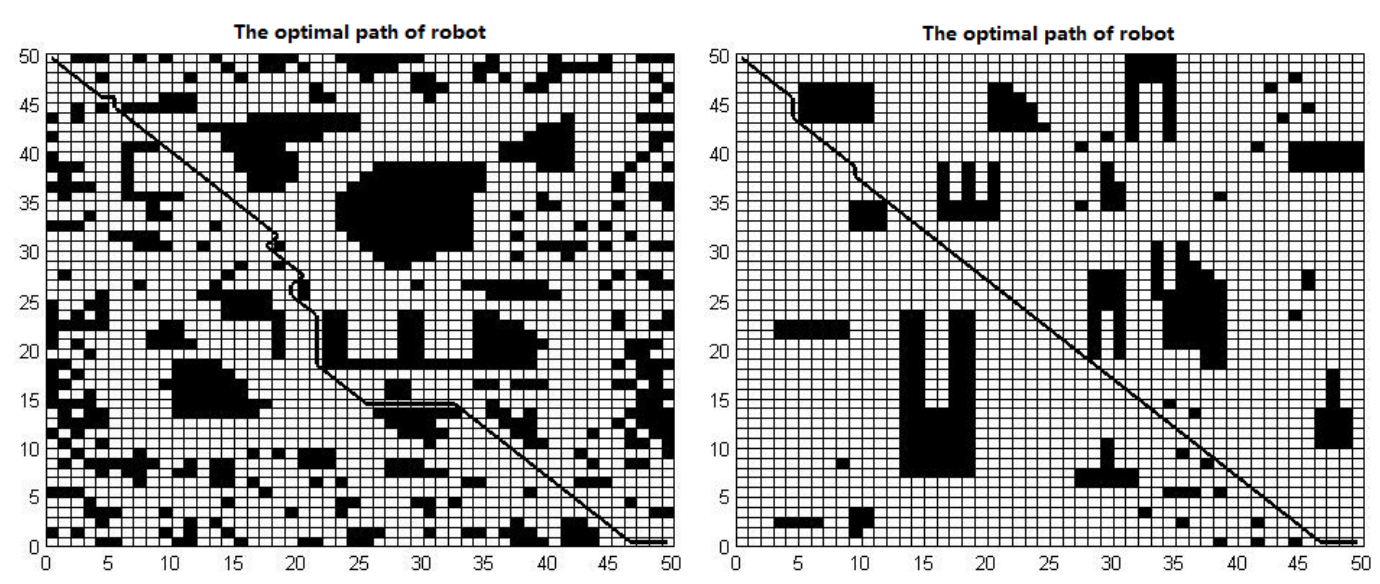

(a) Map $50 \times 50$ Environment 1
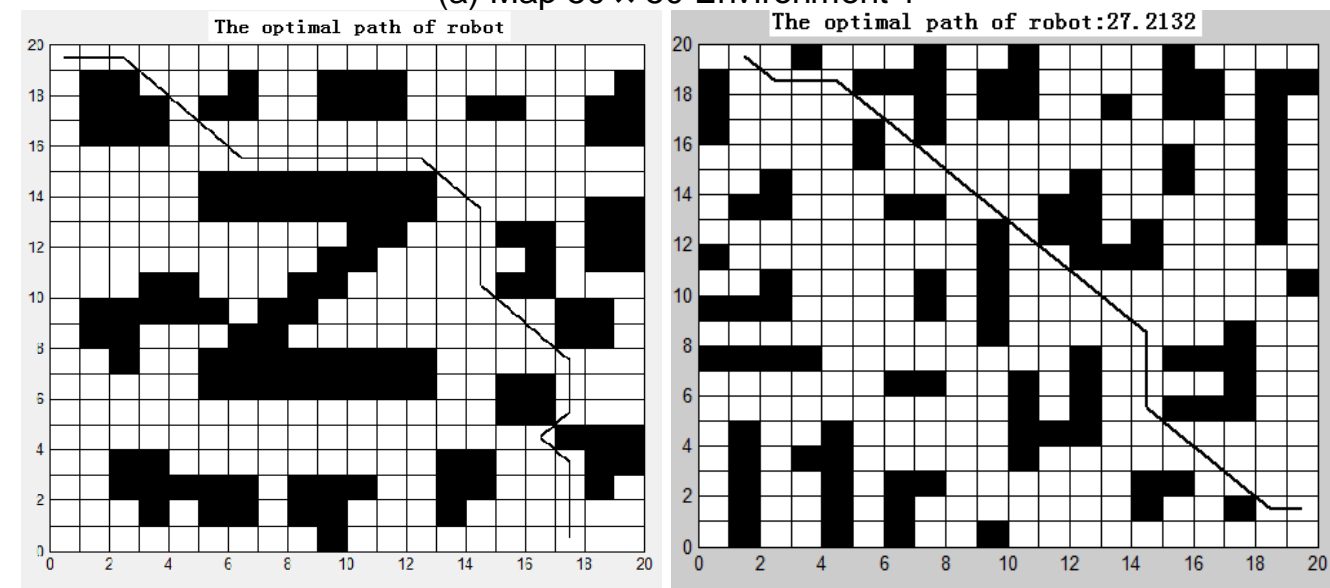

(b) Map $20 \times 20$ Environment 2

Figures 1. Complex Environment, The Solid Line Shows the Path Generated by the HQACO Algorithm

In next section we present an extensive simulation study to evaluate the efficiency of the HQACO algorithm. The parameters of the algorithm are presented in Table 1.

Table 1. Parameter Specification

\begin{tabular}{|l|c|}
\hline Parameters & Val \\
\hline m: number of ants & 20 \\
\hline$\alpha:$ Pheromone trail coefficient & 1 \\
\hline$\beta:$ Heuristic coefficient & 2 \\
\hline $\mathrm{q}_{0}:$ Selection threshold & 0.9 \\
\hline$\theta:$ accelerating convergence factor & 0.9 \\
\hline$\theta_{i}:$ rotate angle & 0.05 \\
\hline$\varphi:$ evaporation trail & 0.2 \\
\hline$\tau_{0}:$ The initial pheromone value & 0.00 \\
\hline $\begin{array}{c}\text { NC: Number of iterations of modified ant } \\
\text { algorithm }\end{array}$ & 200 \\
\hline
\end{tabular}

To verify the convergence rate of HQACO algorithm, we have conducted another 
simulation experiment under the complex environment $220 \times 20$ grids. Figures 2 depicts the convergent curve of HQACO in the grid environment 2 of Figures1 respectively. It costs just a few iterations for HQACO algorithm to convergence.
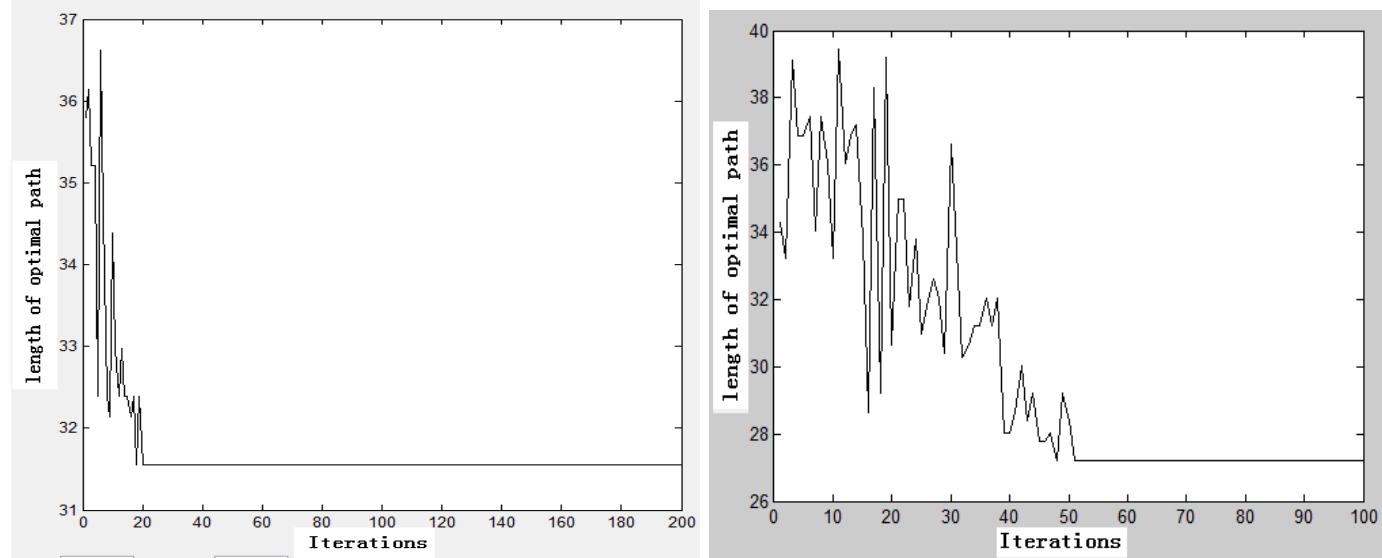

Figures 2. The Convergent Curve of HQACO Algorithm Under the Complex Environment 2 Respectively

For explaining the superiority of HQACO algorithm, we choose improved ant colony algorithm in [13] and our algorithm to test. Figures 3 and Table 2 demonstrate that compared Ref.[13] with our algorithm, our algorithm shows the better solution. In addition, however complex the environment, our algorithm can plan a shorter path quickly.
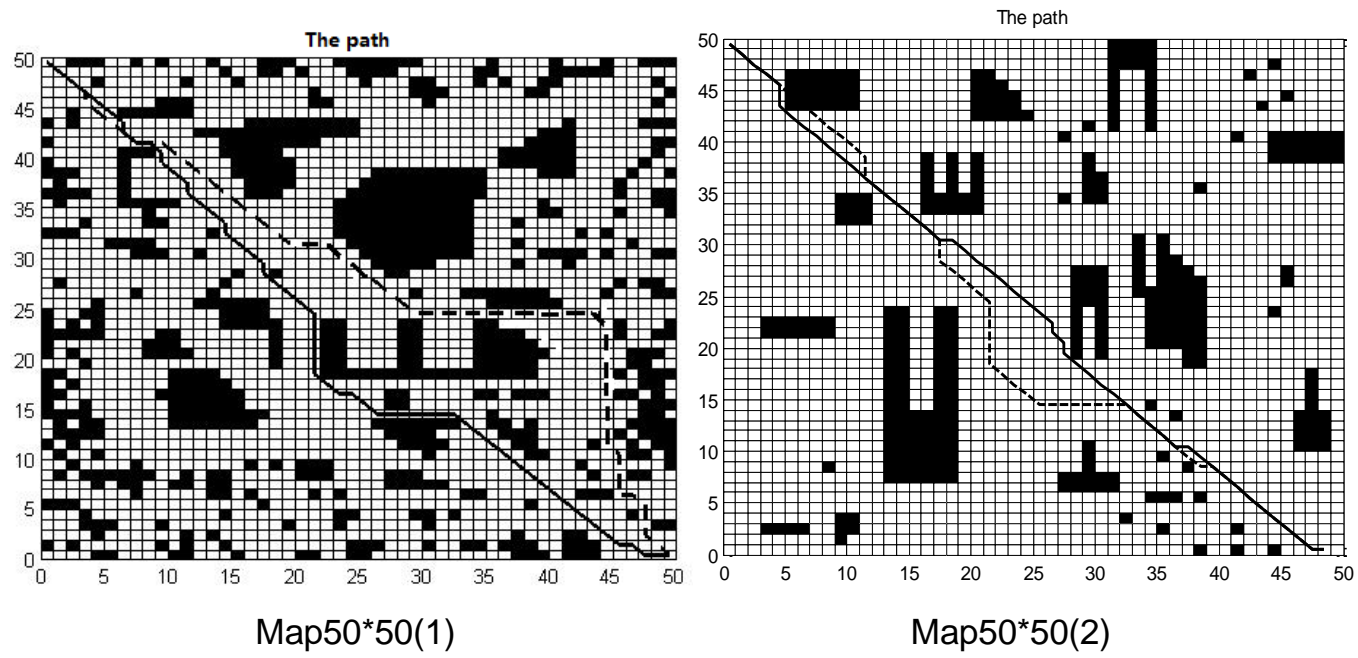

Figures 3. The Solid Line Show the Optimal Path Generated by the HQACO Algorithm Under the Complex Environment 1 Respectively

Table 2. Comparison of Two Algorithm Results

\begin{tabular}{ccc}
\hline algorithm & Path length & Convergence iteration \\
HQACO algorithm (Map50*50(1)) & 75.2 & 47 \\
Ref.[13] algorithm & 79.3 & 60 \\
HQACO algorithm (Map50*50(2)) & 70 & 99 \\
Ref.[13] algorithm & 77.3 & 112 \\
\hline
\end{tabular}

\section{Summary}


In this paper, we proposed a heuristic HQACO Algorithm to solve the global robot path planning under complex environment. The core of the algorithm is that quantum gate is introduced to update the pheromone in ACO, in order to increase the diversity of population. The heuristic operator would balance between population diversity and convergence rate. We presented an extensive simulation study to evaluate our algorithm. As compared with the path planning method in [13], it has been confirmed that the proposed HQACO algorithm has better performance in solution quality and search efficiency. In addition, it can still work out better in the complex environment. The future work is to consider other techniques of establishing environment model for path planning and apply the modified ACO algorithm to the dynamic environment.

\section{Acknowledgements}

This work is partially supported by Natural Science Foundation of China (Grant No. 61272097, 61403249), Innovation Program of Shanghai Municipal Education Commission (Grant No. 12ZZ185), Foundation of No. XKCZ1212. Xiaoming You is corresponding author. The authors also gratefully acknowledge the helpful comments and suggestions of the reviewers, which have improved the presentation.

\section{References}

[1] Imen Chaari, Anis Koubaa, Hachemi Bennaceur, Sahar Trigui and Khaled AI-Shalfan. "smartPATH: A Hybrid ACO-GA Algorithm for Robot Path Planning", 2012 IEEE Congress on Evolutionary Computation, (2012), pp.1-8.

[2] Dorigo M., Gambardella L.M.. "Ant colony system: A cooperative learning approach to the traveling salesman problem",IEEE Trans. Evolutionary Comput. , (1997),pp.53-66.

[3] Silva J. L., Nedjah N., Macedo M. L.and Pessanha F. G.. "Aco-based static routing for network-onchips", in Computational Science and Its Applications ICCSA-2012, ser. Lecture Notes in Computer Science, Springer Berlin Heidelberg, 7333, (2012), pp.113-124.

[4] López-Ibáñez M., Paquete L., Stützle T.. "On the design of ACO for the biobjective quadratic assignment problem”, In: Dorigo, M., Birattari, M., Blum, C., Gambardella, L.M., Mondada, F., Stützle, T. (eds.) ANTS 2004, LNCS, Springer Berlin Heidelberg, 3172,(2004),pp.214-225.

[5] Guanzheng Tan and Dioubate Mamady I."Real-Time Global Optimal Path Planning of Mobile Robots Based on Modified Ant System Algorithm", 2nd International Conference on Natural Computation,4222,(2006),pp.204-214.

[6] Qingbao Zhu. "Ant Algorithm for Path Planning of Mobile Robot in a Complex Environment”, ACTA Automatica Sincia, 32(4), (2006), pp.586-593.

[7] Juan ping Zhao, Xian wen Gao, Xiu hui Fu and Jin gang Liu. "Improved ant colony algorithm of path planning for mobile robot”, Control Theory \& Applications, 28(4), (2011), pp.457-461.

[8] Qi Zhang, Jia cheng Ma, Wei Xie, Li yong Ma, "Improved Ant Colony Algorithm-Based Path Planning for Mobile Robot", Journal of Northeastern University( Natural Science), 34(11), (2013), pp.1521-1524.

[9] Zhe Wang, Shudong Sun, Feixiang Cao. "An Improved Ant Colony Algorithm for Mobile Robot Path Planning under Dynamic Environment", Mechanical Science and Technology for Aerospace Engineering, 32(1), (2013), pp.42-46.

[10] Jiyun Bai and Shiyong Li."Modeling of ant colony foraging behavior based on agent and application of model in robotic path planning", Application Research of Computers, (2013),30.

[11] Xianfeng YANG, Junhui FU. "Mobile Robot Path Planning Based on Grid Algorithm and CGA",Computer Simulation, 29(7), (2012), pp.223-226.

[12] Changan LIU, Xiaohu YAN, Chunyang LIU, "Dynamic Path Planning for Mobile Robot Based on Improved Ant Colony Optimization Algorithm", ACTA ELECTRONICASINICA, 39(5),(2011),pp.1220-1224.

[13] Zheng ZHU, ShiRong LIU, BoTao ZHANG. "Global Path Planning of Mobile Robot Based on Improved Ant Colony Algorithm", Proceedings of the 30th Chinese Control Conference, Yantai,China.(2011),pp.4083-4087.

[14] Ye-Hoon Kim, Jong-Hwan Kim."Multiobjective quantum-inspired evolutionary algorithm for fuzzy path planning of mobile robot”, IEEE Congress on Evolutionary Computation,(2009),pp.1185-1192. 
International Journal of Control and Automation Vol.10, No.4 (2017) 